The 3 poster sessions were well attended with ample opportunity for extended conversations and networking. Themed poster walks, in which attendees were led by a facilitator while presenters shared their research questions, methods, results, and key implications, were held this year. Poster walks offered the opportunity for attendees to learn more about a particular subject matter and research methodologies.

The career of L.J. Fagnan, founding Director of the Oregon Rural Practice-based Research Network and the Meta Learning And Research Consortium (MetaLARC), was celebrated with a lifetime achievement award.

Conference participants were asked to vote for their choice of the best posters for the 2018 David Lanier Poster Awards. Winning posters can be found on the NAPCRG website (http://www.napcrg.org/Conferences/PastMeetingArchives/2018PBRNConference MeetingMaterials).

The enthusiasm and engagement at the 2018 PBRN Conference was high from start to finish. Videos of the 2018 plenary presentations and conference resource materials are available on the NAPCRG website (http:// www.napcrg.org/Conferences/PastMeetingArchives/20 18PBRNConferenceMeetingMaterials).

The 2019 PBRN Conference will take place June 24-25, 2019 in Bethesda with the theme of, "How do we keep prevention on the table in face of disease management incentives?"

Hope to see you there next year!

Donald E. Nease, $J r_{r}, M D_{i}$

Denise Campbell-Scherer, $M D, P b D_{i}$ Jill Haught Funding for this conference was made possible [in part] by 1R13HSO24 893-O2 from the Agency for Healthcare Research and Quality (AHRQ). The views expressed in written conference materials or publications and by speakers and moderators do not necessarily reflect the official policies of the Department of Health and Human Services; nor does mention of trade names, commercial practices, or organizations imply endorsement by the US Government.

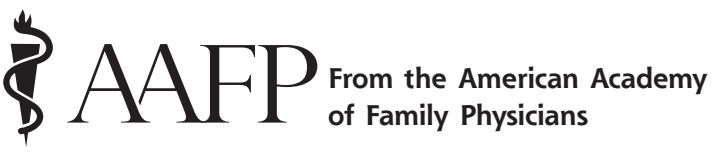

Ann Fam Med 2018;16:471-472. https://doi.org/10.1370/afm.2304.

\section{AAFP OFFERS RESOURCES AS 'JUULING' INCREASES AMONG YOUTH}

One particular brand of e-cigarette, known as JUUL, has been drawing a lot of heat. Extremely popular among adolescents and young adults, the product is the focus of an FDA crackdown and multiple lawsuits.

To ensure that AAFP members stay up-to-date on the concerns swirling around this relative newcomer to the electronic nicotine delivery system (ENDS) market, the Academy has created resources to help educate family physicians (https://www.aafp.org/patient-care/ public-health/tobacco-nicotine/e-cigs.html) and their patients (https://www.aafp.org/dam/AAFP/documents/ patient_care/tobacco/juuling-fact-sheet-patient.pdf) about the product.

\section{What Is JuUL?}

According to former assistant surgeon general and family physician Douglas Kamerow, MD, MPH, of Washington, DC, JUUL's popularity among younger users has driven the product's massive sales, with the device now accounting for almost $70 \%$ of convenience store e-cigarette business.

"It's shaped like a small, sleek USB drive-easy to use and easy to conceal," Kamerow told AAFP News. "High-school and even middle-school students love its shape and ease of use and enjoy flavors such as mango, fruit medley, and cool cucumber."

Each JUUL starter pack contains 4 pods of flavored e-juice that are formulated with nicotine salts from natural tobacco leaves instead of the free-base nicotine commonly used by other e-juice manufacturers, says the Public Health Law Center at Mitchell Hamline School of Law.

"Kids like the flavors and trade them back and forth, as the e-juice pods are removable," said Kamerow. "JUUL has higher nicotine concentrate than other e-cigarettes, so it may be more addictive than other e-cigarette products. There are many anecdotes about kids starting JUUL to be cool but ending up needing to use it every day."

The concentration of nicotine in each JUUL pod is about twice that of many other e-juice nicotine concentrations, listed at $5 \%$ nicotine by weight vs the more common $2.4 \%$ or less in other e-juices.

To combat the perception that JUUL's higher nico- 
tine concentration is leading to more people becoming addicted to the product compared with its rival e-cigarettes, JUUL Labs Inc announced that it will add an option to buy e-juice pods with a $3 \%$ nicotine concentration by weight in the product's mint and Virginia tobacco flavors.

JUUL Labs is also manufacturing reduced-nicotine versions of the product for distribution in the United Kingdom to comply with local regulations, Kamerow said.

What's particularly troubling about nicotine levels in JUULs is a study that found that $63 \%$ of JUUL users aged 15 to 24 years didn't realize the device contains any nicotine, so they wouldn't be aware that they were inhaling an addictive chemical.

Kamerow pointed out that JUULs don't look like classic "cigalike" e-cigarettes, which are round and resemble traditional combustible cigarettes.

"JUUL is not just an e-cigarette in a different package," he explained. "It contains a different type of nicotine, using nicotine salts, that seems to better approximate the mouth feel of cigarettes than other e-cigarettes."

It should also be noted that products similar to JUUL, such as myblu and KandyPens, have also entered the US market.

\section{Center of Controversy}

The FDA said in a statement that its Youth Tobacco Prevention Plan, launched in July 2017, would expand to include several efforts to stop youth use of tobacco products, focusing particularly on e-cigarettes such as JUUL.

These actions included:

- Launching a large-scale, undercover crackdown on the sale of JUUL products to minors by both brickand-mortar and online retailers

- Reaching out to eBay to remove listings for JUUL products on its website, and

- Contacting the product's manufacturer with an official request for information to better understand its marketing efforts; research on the health, toxicological, behavioral or physiologic effects of the product; and how design features, ingredients, or specifications appeal to youth

Following the agency's action, 3 separate lawsuits - 2 filed in California and 1 in New Yorkclaimed the company deceptively marketed the JUUL product as safer than combustible cigarettes and as a viable method to quit smoking. The plaintiffs in these lawsuits contend that "JUULing" has, in fact, proved to be more addictive than using traditional cigarettes and say they're now addicted to the product.

\section{What the Research Shows}

Kamerow noted that despite anecdotes about e-cigarettes helping users to stop smoking combustible cigarettes, there are no good-quality evidence-based studies showing this to be the case. In fact, some studies have shown that these electronic devices not only do not help users quit using tobacco-they may actually increase their odds of smoking.

For example, the National Institute on Drug Abuse found that $30.7 \%$ of teen e-cigarette users started smoking traditional cigarettes within 6 months of use compared to $8.1 \%$ of nonusers who took up smoking.

On the other hand, said Kamerow, there are plenty of nicotine-replacement therapy products that have better research behind them that family physicians can recommend. These include transdermal nicotine patches, nicotine gum, nicotine lozenges, and prescription drugs such as varenicline tartrate (Chantix) and bupropion hydrochloride (Zyban)

\section{AAFP Fact Sheets and Other Resources}

In addition to easy-to-use fact sheets the AAFP has developed for both physicians (https://www.aafp. org/dam/AAFP/documents/patient_care/tobacco/ juuling-fact-sheet-physician.pdf) and patients (https:// www.aafp.org/dam/AAFP/documents/patient_care/ tobacco/juuling-fact-sheet-patient.pdf), the Academy offers members a comprehensive fact sheet on ENDS (https://www.aafp.org/dam/AAFP/documents/ patient_care/tobacco/ends-fact-sheet.pdf), a Tobacco and Nicotine Cessation Toolkit (https://www.aafp.org/ patient-care/public-health/tobacco-nicotine/toolkit. html), and its Ask and Act tobacco cessation program resources (https://www.aafp.org/patient-care/publichealth/tobacco-nicotine/ask-act.html).

One more way family physicians can combat JUUL and ENDS use, said Kamerow, is by asking adolescent patients - and/or telling their parents to ask their adolescent children-whether they "vape" or "JUUL," not just whether they "smoke."

"We need to keep kids from using JUULs and other e-cigarettes, if possible," he urged. "That's why it's so important to educate parents and adolescents about this." Chris Crawford AAFP News 